\title{
Short communication: Antioxidant activity of calf milk replacers
}

\author{
M. A. Soberon, ${ }^{\star 1}$ R. H. Liu, $\dagger$ and D. J. R. Cherney ${ }^{\star}$ \\ ${ }^{*}$ Department of Animal Science, and \\ †Department of Food Science, Cornell University, Ithaca, NY 14853
}

\section{ABSTRACT}

A calf milk replacer (CMR) is designed to replace whole, saleable milk as a lower cost nutrient source for calves while striving to nourish a newborn calf, reduce calf mortality, strengthen immunity, and increase animal life span and productivity. Antioxidants (AO) can enhance immune defense by reducing oxidative damage, but CMR are traditionally not formulated for AO activity. The objective of this study was to compare total AO activities of bovine milk and $6 \mathrm{CMR}$ (A to $\mathrm{F}$ ) that vary in the amount and source of fat and protein. Calf milk replacers were donated by Milk Products LLC (Chilton, WI). Milk was obtained from the Cornell Dairy Research Farm bulk tank, representing milk produced within $24 \mathrm{~h}$ by 455 cows. Milk replacers were mixed to $150 \mathrm{~g} / \mathrm{L}$ with $40^{\circ} \mathrm{C}$ purified water. All samples were extracted in triplicate. Following hexane lipid extraction, both milk and CMR samples were extracted 5 times with ethyl acetate and then evaporated and reconstituted with $70 \%$ methanol:water. Samples were assessed for total AO activity using the peroxyl radical scavenging capacity assay where each sample was diluted to 5 descending concentrations, plated in triplicate. Ascorbic and gallic acids were standards for each plate. Type of protein (soy) had a positive effect on $\mathrm{AO}$ activity for $\mathrm{CMR} \mathrm{A}$, which exhibited the highest total AO activity. Natural bovine milk had the second highest AO activity. Many factors may explain the difference in $\mathrm{AO}$ activity between natural milk and formulated CMR, including fat, vitamin, and mineral contents, enzymatic AO, phenolics, flavonoids, fatty acid profile, and AA composition. When comparing AO activity of CMR, it is important to consider the diversity in feeding recommendations, which will alter the vitamin and mineral content, thus influencing AO activity. The opportunity exists to enhance AO activity of CMR to more closely mimic that of bovine milk. Future research is warranted to compare a broader range of CMR using methods that account for total lipophilic

Received October 27, 2011.

Accepted January 10, 2012.

${ }^{1}$ Corresponding author: mas329@cornell.edu

and hydrophilic $\mathrm{AO}$ activities, as well as to investigate the effect of additional compounds in milk that may affect AO activity.

Key words: antioxidant activity, milk, calf, milk replacer

\section{Short Communication}

Early life nutrition of a calf can potentially affect not only performance and survival during the time of liquid feeding, but also future milk production once the calf reaches adulthood (Soberon et al., 2012). A calf milk replacer (CMR) is designed to mimic the nutritional benefits of bovine milk in an effort to nourish a newborn calf, reduce calf mortality, strengthen immunity, and increase animal life span and productivity. Antioxidants (AO) can enhance immune defense by reducing oxidative damage, which may translate into decreased calf mortality and increased performance. Research in humans has shown that breast milk is significantly higher in AO activity than infant formulas (Friel et al., 2002). However, CMR are not traditionally formulated to take into consideration the dietary $\mathrm{AO}$ consumed by the calf.

Scientific literature regarding AO in bovine milk is targeted toward the effect of lipid peroxidation on milk quality for human consumption rather than potential effects on calf performance. In lactating dairy cows, feeding dietary AO has been found to reduce mastitis (Smith et al., 1984) and common transition disorders that may be caused or exacerbated by oxidative stress (Brzezinska-Slebodzinska et al., 1994; Bradford, 2009).

However, research in humans has also verified the benefits of dietary $\mathrm{AO}$ at times in life when oxidative damage is most severe, such as the first 2 wk postpartum (Friel et al., 2002). The immature immune system of a calf is often exposed to a variety of disease challenges in the first few weeks of life, and the first immune response of an animal during a disease challenge is to generate reactive oxygen metabolites in an effort to kill the bacteria (Dibner et al., 2011). Given that oxidative stress has been associated with gut barrier failure (Dibner et al., 2011), there are obvious negative implications on calf health of having an oxidative 
imbalance. However, dietary AO can be used to reduce the load of peroxide radicals in the diet by reacting with the free radicals to convert them to less reactive compounds (Dibner et al., 2011). This strategy was shown to be effective in reducing the oxidative damage of neonatal ileum tissue in birds (Dibner et al., 1996).

With $69 \%$ of dairy calves in the United States raised on artificial CMR preweaning (USDA, 2007), the opportunity to better fortify postnatal nutrition could greatly affect the dairy industry. Therefore, the objective of this study was to determine the total AO activities of bovine milk and $6 \mathrm{CMR}$, varying in amount and source of fat and protein.

Milk (29\% fat, $29 \%$ CP on a DM basis) was obtained from the Cornell Dairy Research Farm (Harford, NY) bulk tank, representing milk produced within $24 \mathrm{~h}$ by 455 cows and frozen in $89-\mathrm{mL}$ polypropylene vials at $-80^{\circ} \mathrm{C}$. Calf milk replacers representing various fat and protein contents and sources were donated by Milk Products LLC (Chilton, WI). Fat contents of CMR ranged from 15 to $20 \% \mathrm{DM}$ and derived from either animal or vegetable sources (Table 1). Protein ranged from 20 to $28.5 \%$ of DM and was derived from milk, soy, or animal proteins (Table 1). Calf milk replacers were formulated and shipped directly after mixing to ensure that all CMR were fresh, with similar shelf life.

Milk and CMR sample preparation methods were implemented according to R. H. Liu (Cornell University, Ithaca, NY; personal communication). Raw, whole milk samples were thawed in a water bath without light, and centrifuged for $15 \mathrm{~min}$ at $4^{\circ} \mathrm{C}$ and $2,500 \times g$. The upper fat layer was removed and $4 \mathrm{~mL}$ of the skim milk was pipetted into a $50-\mathrm{mL}$ centrifuge tube (Fisher Scientific, St. Louis, MO). All CMR were reconstituted to $150 \mathrm{~g} / \mathrm{L}$ with $40^{\circ} \mathrm{C}$ purified water. Two milliliters of HPLC-grade hexanes (Fisher Scientific) was added to the milk or CMR sample, and the sample was mixed by vortexing for $5 \mathrm{~min}$ and centrifuged for $5 \mathrm{~min}$ at $4^{\circ} \mathrm{C}$ and $2,500 \times g$. The hexane layer was removed to ex- tract lipids and the process was repeated. Samples were acidified to $\mathrm{pH} 2.0$ with $0.5 \mathrm{~mL}$ of $12 \mathrm{M} \mathrm{HCl}$ (Fisher Scientific). Ethyl acetate extraction was performed 5 times, in which $6 \mathrm{~mL}$ of HPLC-grade ethyl acetate was added to the acidified, defatted milk, the sample was vortexed for $5 \mathrm{~min}$ and centrifuged for $5 \mathrm{~min}$ at $4^{\circ} \mathrm{C}$ and $2,500 \times g$, and the ethyl acetate layer collected and pooled. All samples were extracted in triplicate. The ethyl acetate fraction was evaporated to dryness using a rotary vacuum evaporator in a water bath at $46^{\circ} \mathrm{C}$. The sample was reconstituted in $70 \%$ methanol:water and transferred to an amber HPLC vial (Fisher Scientific) and stored at $-40^{\circ} \mathrm{C}$ until analysis.

The AO activity of milk and CMR samples was determined using a peroxyl radical scavenging capacity (PSC) assay described previously (Adom and Liu, 2005; He and Liu, 2006). Each sample was diluted to 5 descending concentrations and plated in triplicate; ascorbic and gallic acids were standards for each plate. The reaction was monitored using the fluorescent dye dichlorolfluorescein. Peroxyl radicals generated by 2,2'-azobis(amidinopropane) (ABAP) oxidize nonfluorescent dichlorofluorescein (DCFH) to fluorescent dichlorofluorescein (DCF). The degree of inhibition of DCFH oxidation by AO that scavenge peroxyl radicals was used as the basis for calculating the AO activity. The reaction mix contained $0.75 M$ phosphate buffer at $\mathrm{pH}$ 7.4, $50 \mathrm{~m} M$ ABAP, $12.5 \mu M \mathrm{DCFH}$ dye, and the appropriate concentrations of the sample extracts. The dye was prehydrolyzed with $1 \mathrm{mM} \mathrm{KOH}$ to remove the diacetate moiety just before use in the reaction, and the reaction was carried out at $37^{\circ} \mathrm{C}$, in a total volume of $200 \mu \mathrm{L}$ using a 96-well plate. Fluorescence generation was monitored (excitation at $485 \mathrm{~nm}$ and emission at $538 \mathrm{~nm}$ ) with a Fluoroskan Ascent fluorescent spectrophotometer (Thermo Labsystems, Franklin, MA). Data were acquired with the Ascent Software, version 2.6 (Thermo Labsystems) running on a personal computer. The areas under the fluorescence reaction time kinetic

Table 1. Antioxidant (AO) activity of bovine milk and calf milk replacers ${ }^{1}$

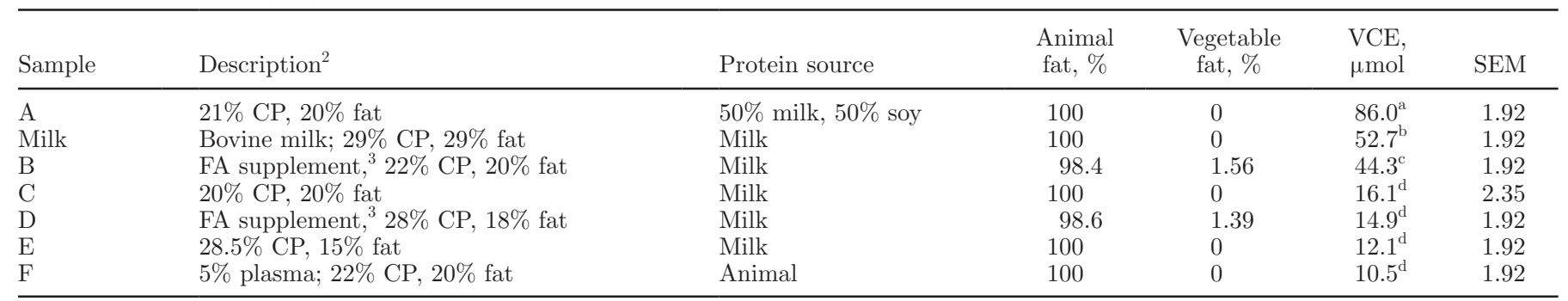

${ }^{\mathrm{a}-\mathrm{d}}$ Means with different superscripts differ, $P<0.01$.

${ }^{1}$ Results for total $\mathrm{AO}$ activity are expressed as $\mu \mathrm{mol}$ of vitamin $\mathrm{C}$ equivalent (VCE)/mL of milk or reconstituted milk replacer.

${ }^{2} \mathrm{CP}$ and fat contents reported on a DM basis.

${ }^{3} \mathrm{FA}$ supplement represents a commercial FA supplement of specific short-, medium-, and long-chain PUFA. 
curve (AUC) for both control and samples were integrated and used as the basis for calculating PSC using the equation $\mathrm{PSC}$ unit $=1-(\mathrm{SA} / \mathrm{CA})$, where $\mathrm{SA}$ is the AUC for the sample or standard dilution and CA is the AUC for the control reaction. Compounds or extracts inhibiting the oxidation of DCFH produced lower SA values and higher PSC values. The parameter $\mathrm{EC}_{50}$ was defined as the dose required to cause $50 \%$ inhibition (PSC value of 0.5 ) for each sample extract and was used as the basis for comparing different compounds or samples. Results obtained for sample extract AO activities were expressed as micromoles of vitamin $\mathrm{C}$ equivalents (VCE) per milliliter of milk or CMR \pm SEM for triplicates.

Data were analyzed statistically using PROC MIXED of SAS 9.2 (SAS Institute Inc., Cary, NC). The effects of fat content, protein content, and CMR on AO activity were independently assessed. Data are reported as least squares means \pm SEM; effects were considered significantly different when $P<0.05$.

The AO activity of defatted natural bovine milk $(52.7 \mu \mathrm{mol}$ of $\mathrm{VCE} / \mathrm{mL})$ analyzed was from cows fed a corn silage-based TMR (Table 1). The AO activity of milk may vary according to the diet of the cow and the processing of the milk (Zulueta et al., 2009).

Although we observed no effect of protein content on AO activity in the samples analyzed, others have reported the casein fraction to be the major contributor to the total AO capacity of whole milk (Zulueta et al., 2009). Although casein may contribute to the AO capacity of whole milk, it is less likely to contribute to the $\mathrm{AO}$ of CMR because the US milk replacer industry now uses whey proteins almost exclusively instead of casein-containing dried skim milk (Lammers et al., 1998). However, the type of protein (soy) had a positive effect on AO activity in the case of CMR A (Table 1 ), which can likely be attributed to the isoflavones and cinnamic acid derivatives present in soy. Despite the contribution of soy protein to $\mathrm{AO}$ activity, its use instead of whey proteins or whole milk in CMR is associated with poorer calf performance due in part to residual antinutritional factors, intestinal abnormalities (Montagne et al., 2000), and differences in AA profile from that of milk protein, (Kanjanapruthipong, 1998).

Although not observed in this study, a potential negative effect of many fat sources in CMR is the addition of high levels of unsaturated fatty acids prone to oxidation, which can be a source of reactive oxygen species (Dibner et al., 2011). The unintentional addition of pro-oxidants via feed ingredients is an area worthy of careful monitoring, as justified by the Novus International Survey from 2000 to 2005 (Dibner et al., 2011). Novus reported that 40 to $50 \%$ of the fat sold for use in animal feeds was unstable, with even higher percentages of instability observed in warmer months of the year (Dibner et al., 2011). Thus, although the CMR analyzed in this study were shipped from the manufacturer directly after mixing and subsequently stored at $-20^{\circ} \mathrm{C}$ until analysis, CMR used on farms will be more susceptible to the effects of storage conditions and feed ingredient quality on AO activity.

The AO activity of bovine milk was significantly higher than that of the CMR with the exception of CMR A (Table 1). The difference in AO activity of bovine milk may be due in part to its AA composition (Clausen et al., 2009) and increased fat content (Chen et al., 2003), which can influence the concentration of carotenoids (Lindmark-Månsson and Akesson, 2000). Moreover, the vitamin content of milk and CMR often differ. Hydrophilic compounds such as vitamin $\mathrm{C}$ and uric acid were the largest contributors to the total AO capacity of deproteinized milk according to Zulueta et al. (2009). Ascorbic acid (vitamin C) concentrations in natural bovine milk range from 5.9 to $14.0 \mathrm{mg} / \mathrm{L}$ (Lindmark-Månsson and Akesson, 2000) but many CMR do not formulate for ascorbic acid.

In addition, the FA profile of whole milk differed significantly from that of most of the CMR studied, which typically lacked short- and medium-chain FA. The FA profiles of some CMR, such as B and D, better matched the FA profile of milk through the addition of a commercial FA supplement containing specific short-, medium-, and long-chain PUFA. Calf milk replacers $\mathrm{B}$ and $\mathrm{D}$ had different AO activity $(P<0.01)$ when the comparison was made based on the concentration of $150 \mathrm{~g}$ of $\mathrm{CMR} / \mathrm{L}$ of water. However, at this concentration, CMR D had roughly half the amount of vitamin and trace mineral premix (and, therefore, half the levels of vitamins $\mathrm{A}, \mathrm{D}$, and E) because the feeding recommendation is $1.02 \mathrm{~kg} / \mathrm{d}$, whereas CMR B is formulated to be fed at $0.57 \mathrm{~kg} / \mathrm{d}$. If $\mathrm{AO}$ activity had been compared based upon total AO fed rather than concentration, then CMR B and D may not have differed significantly in AO activity. Only one other CMR (E) had this increased feeding recommendation, as is customary for higher protein CMR designed for accelerated growth; all others were formulated to be fed at $0.57 \mathrm{~kg} / \mathrm{d}$. Therefore, the AO activity of CMR B (and likely $\mathrm{D}$ if the evaluation was made based on total AO fed), although still lower than that of milk, was higher than that of the other CMR, possibly because of this FA supplement.

Although fat sources in CMR may present an unintentional source of pro-oxidants if the quality of the ingredients and storage conditions are compromised, when milks with varying fat contents were analyzed for total AO activity, others reported a small contribution to total AO activity from the liposoluble AO compo- 
nents of whole milk versus skim milk (Zulueta et al., 2009). Although fat content tended to have an effect ( $P$ $=0.057$ ) on $\mathrm{AO}$ activity in the samples analyzed, losses in lipid-bound AO were not fully accounted for because the milk and CMR were defatted before analysis. Future research is warranted to compare CMR with a broader range of FA content and profiles using methods that account for total lipophilic and hydrophilic AO.

The AO activity of milk is the result of the combination of native antioxidative enzymes as well as nonenzymatic $\mathrm{AO}$ such as lactoferrin, vitamins $\mathrm{C}$ and $\mathrm{E}$, phenolics and flavonoids, and fat-soluble carotenoids (Lindmark-Månsson and Akesson, 2000). In CMR, the AO activity is dependent upon the inclusion of nonenzymatic AO supplied as essential nutrients, most commonly vitamin $\mathrm{E}$ and selenium, and occasionally vitamin C.

Overall, the opportunity exists to enhance AO activity of CMR to more closely mimic the AO activity of bovine milk. Despite exhibiting the highest total AO activity, CMR with a high percentage of soy protein are generally not recommended because of the lower performance associated with soy protein. Natural bovine milk had the second highest AO activity. Fat, vitamin and mineral contents, enzymatic $\mathrm{AO}$, phenolics and flavonoids, FA profile, and AA composition are among the many factors that may explain the difference in $\mathrm{AO}$ activity between natural milk and formulated CMR. When comparing the $\mathrm{AO}$ activity of CMR, it is important to consider the diversity in feeding recommendations, which will alter the vitamin and mineral content, and thus influence AO activity. Strategies for increasing the $\mathrm{AO}$ activity of $\mathrm{CMR}$ should include ensuring the use of high-quality ingredients, proper storage of CMR, and the inclusion of dietary $\mathrm{AO}$ as essential nutrients.

\section{ACKNOWLEDGMENTS}

The authors thank Milk Products LLC (Chilton, WI) for the donation of CMR used in the study and David Carlson and Patrick Kahle for coordinating the donation, the Department of Animal Science at Cornell University (Ithaca, NY) for financial and academic support, as well as Richard Austic, Ron Butler, Bruce Berggren-Thomas, Chris DeRito, Luciana Malta, Thomas Overton, Susanne Pelton, and Fernando Soberon (of Cornell University) for analytical support.

\section{REFERENCES}

Adom, K. K., and R. H. Liu. 2005. Rapid peroxyl radical scavenging capacity (PSC) assay for assessing both hydrophilic and lipophilic antioxidants. J. Agric. Food Chem. 53:6572-6580.

Bradford, B. J. 2009. Inflammation and transition cow disorders. Page 76-81 in Proc. Four State Dairy Nutrition and Management Conference, Dubuque, IA.

Brzezinska-Slebodzinska, E., J. K. Miller, J. D. Quigley III, and J. R. Moore. 1994. Antioxidant status of dairy cows supplemented prepartum with vitamin E and selenium. J. Dairy Sci. 77:3087-3095.

Chen, J., H. Lindmark-Mansson, L. Gorton, and B. Akesson. 2003 Antioxidant capacity of bovine milk as assayed by spectrophotometric and amperometric methods. Int. Dairy J. 13:927-935.

Clausen, M. R., L. H. Skibsted, and J. Stagsted. 2009. Characterization of major radical scavenger species in bovine milk through size exclusion chromatography and functional assays. J. Agric. Food Chem. 57:2912-2919.

Dibner, J. J., C. Atwell, M. Kitchell, W. Shermer, and F. Ivey. 1996. Feeding of oxidized fats to broilers and swine: Effects on enterocyte turnover, hepatocyte proliferation and the gut associated lymphoid tissue. Anim. Feed Sci. Technol. 62:1-13.

Dibner, J. J., M. Vazquez-Anon, and C. D. Knight. 2011. Understanding oxidative balance and its impact on animal performance. Page 1 in Proc. Cornell Nutrition Conference for Feed Manufacturers, Cornell University, Syracuse, NY.

Friel, J. K., S. M. Martin, M. Langdon, G. R. Herzberg, and G. R. Buettner. 2002. Milk from mothers of both premature and fullterm infants provides better antioxidant protection than does infant formula. Pediatr. Res. 51:612-618.

He, X., and R. H. Liu. 2006. Cranberry phytochemicals: Isolation, structure elucidation, and their antiproliferative and antioxidant activities. J. Agric. Food Chem. 54:7069-7074.

Kanjanapruthipong, J. 1998. Supplementation of milk replacers containing soy protein with threonine, methionine, and lysine in the diets of calves. J. Dairy Sci. 81:2912-2915.

Lammers, B. P., A. J. Heinrichs, and A. Aydin. 1998. The effect of whey protein concentrate or dried skim milk in milk replacer on calf performance and blood metabolites. J. Dairy Sci. 81:19401945.

Lindmark-Månsson, H., and B. Akesson. 2000. Antioxidative factors in milk. Br. J. Nutr. 84:S103-S110.

Montagne, L., R. Toullec, M. Formal, and J. P. Lalles. 2000. Influence of dietary protein level and origin on the flow of mucin along the small intestine of the preruminant calf. J. Dairy Sci. 83:28202828.

Smith, K. L., J. H. Harrison, D. D. Hancock, D. A. Todhunter, and H. R. Conrad. 1984. Effect of vitamin E and selenium supplementation on incidence of clinical mastitis and duration of clinical symptoms. J. Dairy Sci. 67:1293-1300.

Soberon, F., E. Raffrenato, R. W. Everett, and M. E. Van Amburgh. 2012. Pre-weaning milk replacer intake and effects on long-term productivity of dairy calves. J. Dairy Sci. 95:783-793.

USDA. 2007. Part I: Reference of Dairy Health and Management in the United States. National Animal Health Monitoring System, Fort Collins, CO

Zulueta, A., A. Maurizi, A. Frigola, M. J. Esteve, R. Coli, and G. Burini. 2009. Antioxidant capacity of cow milk, whey and deproteinized milk. Int. Dairy J. 19:380-385. 